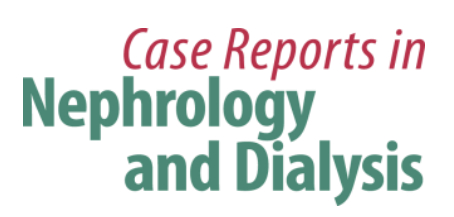

Case Rep Nephrol Dial 2016;6:114-119

\title{
Reversal of Gastric Bypass Resolves Hyperoxaluria and Improves Oxalate Nephropathy Secondary to Roux-en-Y Gastric Bypass
}

\author{
Varun Agrawal $^{\mathrm{a}} \quad$ Jonathan B. Wilfong ${ }^{\mathrm{b}} \quad$ Christopher E. Rich \\ Pamela C. Gibson ${ }^{d}$ \\ aDivision of Nephrology and Hypertension, University of Vermont, Burlington, Vt., USA; \\ ${ }^{b}$ Department of Medicine, University of Vermont, Burlington, Vt., USA; ${ }^{c}$ Bozeman Health \\ Nephrology, Bozeman, Mont., USA; ${ }^{d}$ Department of Pathology and Laboratory Medicine, \\ University of Vermont, Burlington, Vt., USA
}

\section{Keywords}

Roux-en-Y gastric bypass - Hyperoxaluria - Oxalate nephropathy · Acute kidney injury · Reversal of gastric bypass

\begin{abstract}
Hyperoxaluria after Roux-en-Y gastric bypass (RYGB) increases the risk for kidney injury. Medical therapies for hyperoxaluria have limited efficacy. A 65-year-old female was evaluated for acute kidney injury [AKI, serum creatinine $(\mathrm{Cr}) 2.1 \mathrm{mg} / \mathrm{dl}$, baseline $\mathrm{Cr} 1.0 \mathrm{mg} / \mathrm{dl}$ ]. She did not have any urinary or gastrointestinal symptoms or exposure to nephrotoxic agents. Sixteen months prior to this evaluation, she underwent RYGB for morbid obesity. Her examination was unremarkable for hypertension or edema and there was no protein or blood on urine dipstick. Kidney biopsy revealed acute tubulointerstitial nephritis with oxalate crystals in tubules. The concurrent finding of severe hyperoxaluria (urine oxalate $150 \mathrm{mg} /$ day) confirmed the diagnosis of oxalate nephropathy. Despite medical management of hyperoxaluria, her AKI worsened. Laparoscopic reversal of RYGB was performed and within 1 month, her hyperoxaluria resolved (urine oxalate $20 \mathrm{mg} /$ day) and AKI improved ( $\mathrm{Cr} 1.7 \mathrm{mg} / \mathrm{dl})$. Surgical reversal of RYGB may be considered in patients with oxalate nephropathy at high risk of pro-
\end{abstract}




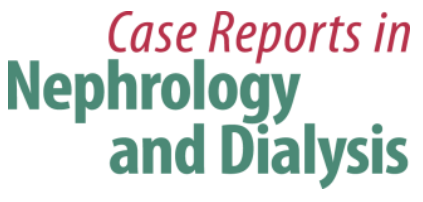

Case Rep Nephrol Dial 2016;6:114-119

DOI: 10.1159/000449128

C 2016 The Author(s). Published by S. Karger AG, Basel www.karger.com/cnd

Agrawal et al.: Reversal of Gastric Bypass Resolves Hyperoxaluria and Improves Oxalate Nephropathy Secondary to Roux-en-Y Gastric Bypass

gression who fail medical therapy. Physicians need to be aware of the possibility of oxalate nephropathy after RYGB and promptly treat the hyperoxaluria to halt further kidney damage.

(C) 2016 The Author(s)

Published by S. Karger AG, Basel

\section{Introduction}

Increased urinary oxalate excretion is a rare cause of acute kidney injury (AKI) that can potentially lead to end-stage kidney disease. We present an interesting case report of a patient with oxalate-mediated renal damage that illustrates the efficacy of a novel therapeutic option to halt the loss of kidney function.

\section{Case Report}

A 65-year-old Caucasian female was referred to the nephrology clinic for decreased kidney function [serum creatinine $(\mathrm{Cr}) 2.1 \mathrm{mg} / \mathrm{dl}$, estimated glomerular filtration rate (eGFR) $24 \mathrm{ml} / \mathrm{min} / 1.73 \mathrm{~m}^{2}$; baseline $\mathrm{Cr} 1.0 \mathrm{mg} / \mathrm{dl}$, eGFR $59 \mathrm{ml} / \mathrm{min} / 1.73 \mathrm{~m}^{2} 5$ months prior to the visit]. She denied fever, rash, arthralgia, gastrointestinal or urinary complaints. There was no known exposure to nephrotoxic agents. She had undergone laparoscopic Roux-en-Y gastric bypass (RYGB) surgery 16 months ago for morbid obesity (body mass index 43.9) and type 2 diabetes mellitus (hemoglobin $A_{1 c}$ of $7.9 \%$ ). RYGB was performed in the standard fashion (Roux limb was $100 \mathrm{~cm}$ long) following which she lost $18.2 \mathrm{~kg}$ in the first 2 months and her glycemic control improved. She had had diabetes mellitus for the past 16 years with no known end-organ damage and Crohn's disease for 20 years, but no disease activity was seen on a recent colonoscopy. Her home medications included multivitamins (containing vitamin C $100 \mathrm{mg} /$ day), iron, omeprazole, pravastatin and venlafaxine (benazepril and metformin were discontinued prior to nephrology evaluation) and there was no family history of kidney disease. Her examination was unremarkable for hypertension (blood pressure $132 / 69 \mathrm{~mm} \mathrm{Hg}$ ) or edema.

Urine studies revealed pH 5.5, specific gravity 1.020 and no protein or blood on urine dipstick. Fractional excretion of sodium in this urine sample was $1.4 \%$, while her blood urea nitrogen was $21 \mathrm{mg} / \mathrm{dl}$ and her hemoglobin $\mathrm{A}_{1 \mathrm{c}}$ level was 6.8\%. Renal ultrasound showed kidneys of normal size and echogenicity with one small nonobstructing stone in each kidney. Kidney biopsy was performed for unexplained AKI. Light microscopy showed acute tubular injury with clear crystalline material scattered in the tubular lumens and the interstitium that was strongly polarizable (fig. 1a, b). Mild lymphocytic interstitial inflammation involving $20 \%$ of the renal cortex and no significant glomerular damage were seen. A diagnosis of oxalate nephropathy presumed secondary to fat malabsorption after RYGB was made. A 24hour urine study at this time was notable for severe hyperoxaluria (urine oxalate 150 $\mathrm{mg} /$ day; normal 4-31 mg/day).

Low oxalate and fat diet, high fluid intake (2.5-3 l/day), calcium carbonate $(500 \mathrm{mg}$ three times daily with each meal) and potassium citrate (30 meq daily) were promptly started and uptitrated as tolerated (calcium carbonate 1,000 mg three times daily with each meal and potassium citrate 70 meq daily). However, the severe hyperoxaluria and AKI persisted (fig. 2). Because of the concern for rapid progression to kidney failure, the decision was made to pursue laparoscopic reversal of RYGB. In this procedure, the small gastric pouch was reattached to the remnant stomach and the Roux limb was resected. One month after 


\section{Case Reports in Nephrology and Dialysis}

surgical reversal, hyperoxaluria resolved (urine oxalate $20 \mathrm{mg}$ /day) and AKI improved ( $\mathrm{Cr}$ $1.7 \mathrm{mg} / \mathrm{dl}$ ). Eight months later, these changes persisted, but she was noted to have a $16.3-\mathrm{kg}$ weight gain and worsening glycemic control (hemoglobin $A_{1 c} 7.2 \%$ ).

\section{Discussion}

Hyperoxaluria (urine oxalate $\geq 45 \mathrm{mg} /$ day) is an adverse effect of RYGB that can cause kidney injury in the form of oxalate nephropathy or calcium oxalate nephrolithiasis. The incidence of hyperoxaluria after RYGB among non-stone formers is reported to be $42-67 \%$ at 1-3.5 years of follow-up [1-4]. Oxalate nephropathy, as proven by kidney biopsy, portends a poor prognosis as prolonged deposition of oxalate crystals in the renal tubules causes tubular damage, interstitial fibrosis and progression of kidney disease. In a case series of biopsy-proven oxalate nephropathy after RYGB $(n=11)$, Nasr et al. [5] reported that the diagnosis was made at a median of 12 months after the surgery and $73 \%$ progressed to endstage renal disease in 3 months. These findings highlight the need to recognize hyperoxaluria as a complication of RYGB and promptly institute therapeutic measures aimed at reducing urine oxalate and arresting kidney damage. While Crohn's disease and excessive vitamin $C$ intake can also cause hyperoxaluria, our patient had a normal colonoscopy and her vitamin $\mathrm{C}$ dose $(100 \mathrm{mg} /$ day) was considerably less than the $1,000 \mathrm{mg} /$ day dose that has been reported to cause hyperoxaluria [5].

Fat malabsorption after RYGB occurs as a result of the dietary fat bypassing the duodenum and jejunum where usually its degradation by pancreatic enzymes and bile salts takes place [6]. The undigested fatty acids bind to intraluminal calcium in the small intestine, thus leaving a large load of unbound oxalate that is subsequently absorbed in the colon leading to hyperoxaluria. Medical management of hyperoxaluria after RYGB focuses on lowering dietary fat and oxalate intake, using enteric oxalate-binding agents (calcium carbonate or calcium citrate with food), use of bile acid sequestrant (cholestyramine) or probiotics such as Oxalobacter formigenes or Lactobacillus sp. that can degrade intraluminal oxalate [7]. Implementation of these measures requires patient compliance and the efficacy may be variable.

To our knowledge, ours is the first case report to demonstrate resolution of enteric hyperoxaluria and clinical improvement of renal function by reversal of RYGB. Our novel approach to a rare but challenging renal complication has important implications for internists, surgeons and nephrologists caring for patients after RYGB. While the degree of hyperoxaluria after RYGB is variable, progressive loss of GFR in our patient with severe hyperoxaluria despite medical management prompted us to consider reversal of RYGB. The resolution of hyperoxaluria after reversal of RYGB was durable and was seen likely as fat malabsorption ceased to occur. Similar findings were observed with reversal of jejunoileal bypass, an obsolete bariatric surgery that achieved weight loss through intense malabsorption [8]. Our patient's eGFR did not return back to the baseline level, but we believe that stopping the decline in eGFR with modest improvement in her renal function was an excellent outcome. Bariatric surgeons report reversal of RYGB to be safe and postoperative complications are minimal [9]. Weight gain and recurrence of obesity-associated comorbidities (such as diabetes) are predictable consequences. Following reversal of RYGB, other surgical options for weight loss such as vertical sleeve gastrectomy or gastric band can be offered that induce weight loss primarily through gastric restriction and have less risk of causing hyperoxaluria [10]. 
In conclusion, hyperoxaluria is a common adverse effect of RYGB that can cause renal damage. Our case demonstrates that a patient presenting with AKI and a history of RYGB should be evaluated for oxalate nephropathy and nephrolithiasis. Definitive diagnosis of oxalate nephropathy is made by kidney biopsy and the characteristic finding of oxalate crystals in renal tubules with associated inflammatory injury. We recommend prompt initiation of measures to reduce 24-hour urine oxalate and addressing other lithogenic factors (such as decreased urine volume and citrate) [7]. Reversal of RYGB to halt kidney injury may be considered in patients with progressive loss of kidney function due to proven oxalate nephropathy, especially if medical therapies fail to reduce hyperoxaluria.

\section{Acknowledgements}

We thank Sadik Sharef, MD, Division of Nephrology and Hypertension, University of Vermont College of Medicine, Burlington, Vt., USA, for performing the kidney biopsy and Michael A. Hill, MD, Adirondack Surgical Group, Plattsburgh, N.Y., USA, for the surgical procedures. This case report was presented as a poster at the American College of Physicians 2015 Vermont Chapter Meeting and at the American College of Physicians Internal Medicine Meeting 2016.

\section{Statement of Ethics}

Consent for publication was obtained from the patient.

\section{Disclosure Statement}

The authors have no conflicts of interest to declare.

\section{References}

1 Sinha MK, et al: Hyperoxaluric nephrolithiasis is a complication of Roux-en-Y gastric bypass surgery. Kidney Int 2007;72:100-107.

-2 Duffey BG, et al: Hyperoxaluria is a long-term consequence of Roux-en-Y Gastric bypass: a 2-year prospective longitudinal study. J Am Coll Surg 2010;211:8-15.

-3 Nelson WK, Houghton SG, Milliner DS, Lieske JC, Sarr MG: Enteric hyperoxaluria, nephrolithiasis, and oxalate nephropathy: potentially serious and unappreciated complications of Roux-en-Y gastric bypass. Surg Obes Relat Dis 2005;1:481-485.

-4 Maalouf NM, Tondapu P, Guth ES, Livingston EH, Sakhaee K: Hypocitraturia and hyperoxaluria after Roux-en-Y gastric bypass surgery. J Urol 2010;183:1026-1030.

5 Nasr SH, et al: Oxalate nephropathy complicating Roux-en-Y gastric bypass: an underrecognized cause of irreversible renal failure. Clin J Am Soc Nephrol 2008;3:1676-1683.

-6 Kumar R, et al: Fat malabsorption and increased intestinal oxalate absorption are common after Rouxen-Y gastric bypass surgery. Surgery 2011;149:654-661.

-7 Agrawal V, et al: Calcium oxalate supersaturation increases early after Roux-en-Y gastric bypass. Surg Obes Relat Dis 2014;10:88-94.

8 Hassan I, Juncos LA, Milliner DS, Sarmiento JM, Sarr MG: Chronic renal failure secondary to oxalate nephropathy: a preventable complication after jejunoileal bypass. Mayo Clin Proc 2001;76:758-760.

-9 Campos GM, Ziemelis M, Paparodis R, Ahmed M, Davis DB: Laparoscopic reversal of Roux-en-Y gastric bypass: technique and utility for treatment of endocrine complications. Surg Obes Relat Dis 2014;10:36-43.

10 Penniston KL, Kaplon DM, Gould JC, Nakada SY: Gastric band placement for obesity is not associated with increased urinary risk of urolithiasis compared to bypass. J Urol 2009;182:2340-2346. 


\section{Case Reports in Nephrology and Dialysis}

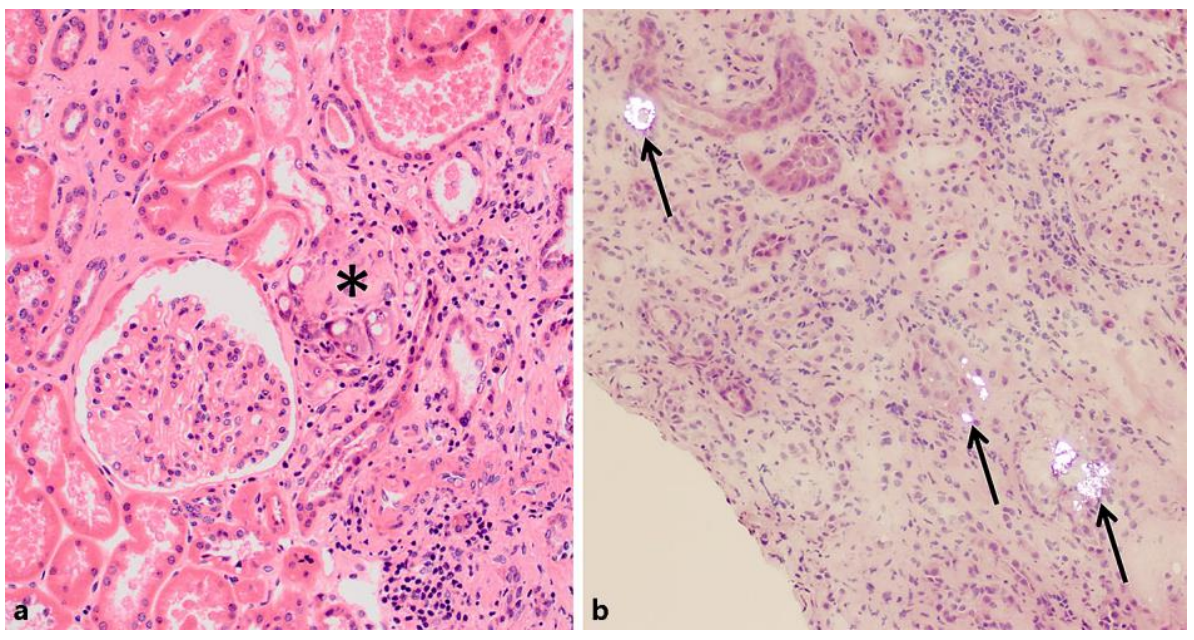

Fig. 1. a, b Kidney biopsy, light microscopy, hematoxylin and eosin stain. a Standard illumination (original magnification, $\times 200$ ) demonstrating tubulo-interstitial injury with giant cell reaction (marked with an asterisk) and mixed interstitial inflammation. b Under polarized light (original magnification, $\times 100$ ), bright polarizable intratubular and interstitial crystals (marked with arrows) were seen that were clear and colorless under bright light. 


\section{Case Reports in Nephrology and Dialysis}

\begin{tabular}{c|c|c|c|c}
\hline Event & $\begin{array}{c}\text { Initial } \\
\text { evaluation by } \\
\text { Nephrology }\end{array}$ & $\begin{array}{c}\text { Following } \\
\text { medical } \\
\text { therapy for } \\
\text { hyperoxaluria }\end{array}$ & $\begin{array}{c}1 \text { month after } \\
\text { reversal of } \\
\text { RYGB }\end{array}$ & $\begin{array}{c}9 \text { months after } \\
\text { reversal of } \\
\text { RYGB }\end{array}$ \\
\hline $\begin{array}{c}\text { Time after } \\
\text { RYGB }\end{array}$ & 16 months & 17 months & 20 months & 28 months
\end{tabular}
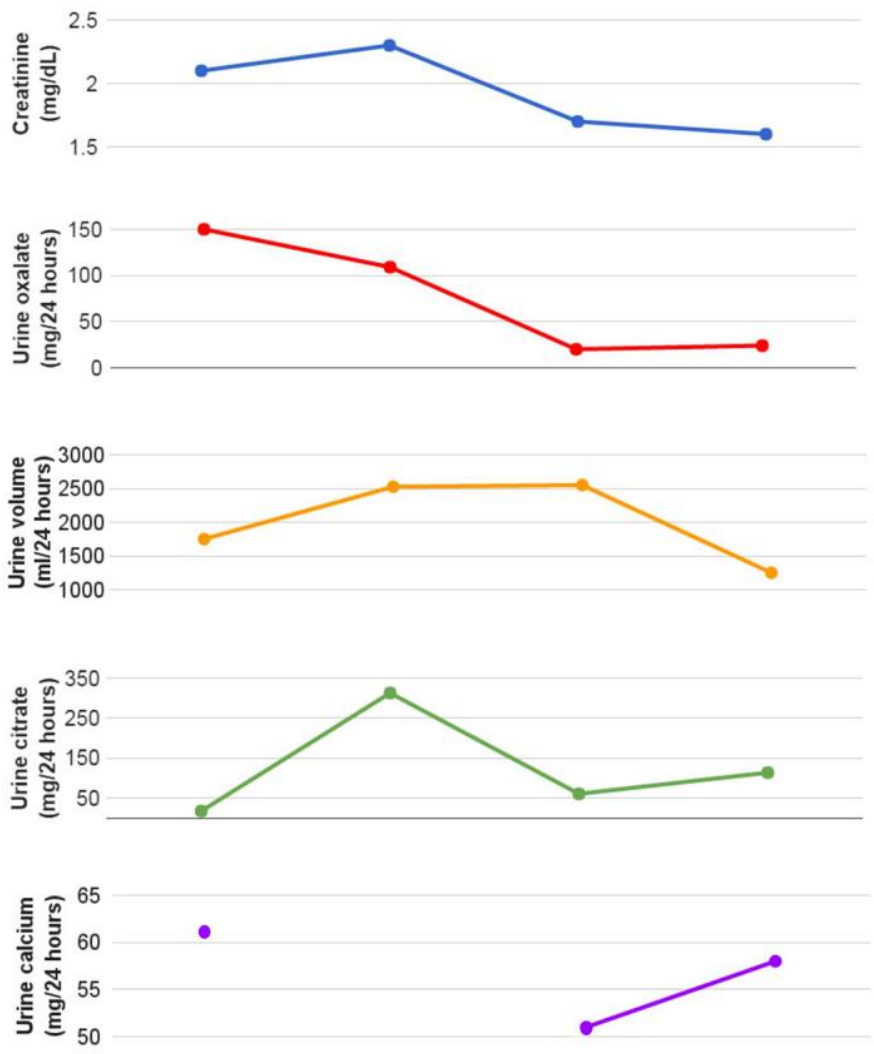

Fig. 2. Changes in renal function and urine parameters in the patient. Urine calcium was not measured in the second 24-hour urine collection. Normal range for urine oxalate: 4-31 mg/24 h, urine citrate: $320-$ $1,240 \mathrm{mg} / 24 \mathrm{~h}$, urine calcium: $100-300 \mathrm{mg} / 24 \mathrm{~h}$. 